\title{
Pressure anisotropy and small spatial scales induced by velocity shear
}

\author{
D. Del Sarto* \\ Institut Jean Lamour, UMR CNRS 7198 and Université de Lorraine, B.P. 70239, F-54506 Vandouvre-lès-Nancy Cedex, France \\ F. Pegoraro and F. Califano \\ Physics Department and CNISM, University of Pisa, 56216 Pisa, Italy \\ (Received 1 July 2015; revised manuscript received 11 April 2016; published 5 May 2016)
}

\begin{abstract}
By including the full pressure tensor dynamics in a fluid plasma model, we show that a sheared velocity field can provide an effective mechanism that makes the initial isotropic pressure nongyrotropic. This is distinct from the usual gyrotropic anisotropy related to the fluid compressibility and usually accounted for in doubleadiabatic models. We determine the time evolution of the pressure agyrotropy and discuss how the propagation of "magnetoelastic perturbations" can affect the pressure tensor anisotropization and its spatial filamentation, which are due to the action of both the magnetic field and the flow strain tensor. We support this analysis with a numerical integration of the nonlinear equations describing the pressure tensor evolution.
\end{abstract}

DOI: 10.1103/PhysRevE.93.053203

\section{INTRODUCTION}

The aim of this article is to show that a sheared velocity field in a weakly collisional, magnetized plasma drives a macroscopic pressure anisotropization in the plane of the velocity strain tensor. This represents a general mechanism when collisional relaxation is either absent or slow that causes part of the kinetic energy of the plasma flow to be locally transformed into anisotropic "internal energy." This energy conversion implies that shear flows do not affect the plasma dynamics only through the fluid destabilization of Kelvin-Helmholtz (KH) modes [1] but can lead to the onset of additional phase-space instabilities driven by the induced pressure anisotropy. In addition, this mechanism provides a possible explanation of the nongyrotropic distribution functions often measured in astrophysical plasmas.

In magnetized plasmas the fast particle gyromotion in a sufficiently strong field makes the pressure tensor $\Pi_{i j}$ isotropic in the plane perpendicular to the magnetic-field direction but allows for different parallel and perpendicular pressures (gyrotropic anisotropy $\hat{A}^{\mathrm{gyr}} \equiv \Pi_{\|} / \Pi_{\perp}$, as is the case for the double-adiabatic or CGL [2] closure). Here we show how the gradient tensor $\partial u_{i} / \partial x_{j}$ acting on $\Pi_{i j}$ has a twofold effect. First, through its rotational component it combines or competes with the gyrotropic effect due to the magnetic field; second, through its incompressible rate of shear (its symmetrical traceless component), it induces pressure agyrotropy (nongyrotropic pressure), quantified as $\hat{A}^{\text {n.g. }} \equiv$ $\left(\Pi_{1}-\Pi_{2}\right) /\left(\Pi_{1}+\Pi_{2}\right)$, with 1 and 2 labeling the principal axes of the local pressure tensor in the plane perpendicular to the magnetic field (taken to coincide with the velocity shear plane). We discuss in particular the role of the rate of shear in the nongyrotropic, full pressure tensor dynamics as obtained from the second moment of the Vlasov equation and its interplay with both the gyrotropic dynamics induced by the magnetic field and fluid vorticity and the propagation of magnetoelastic perturbations [3].

Note that, in fluid dynamics, turbulence is termed "anisotropic" in a different sense from the velocity-space

\footnotetext{
*daniele.del-sarto@univ-lorraine.fr
}

anisotropy meant here, namely, when the velocity field is not statistically invariant under rotations and reflections, and it is known how this symmetry is broken, e.g., by a KH-unstable velocity shear [4] or by a Von Karman flow [5]. On the other hand, in magnetized plasmas pressure anisotropy is mostly meant as gyrotropic in the CGL sense [6]. Although the model we discuss also accounts for gyrotropic anisotropy, as first shown in Ref. [7], which is due to compressibility effects when heat transfer mechanisms are disregarded, here we focus on a possible explanation of the source of the nongyrotropic pressure anisotropy.

This mechanism can affect both the onset and the development of shear-induced fluid instabilities (e.g., $\mathrm{KH}$ ) in plasmas and of anisotropic turbulence and is relevant to the understanding of the origin of some of the non-Maxwellian states, evidenced both in Vlasov simulations [8,9] and in experiments [10-21], occasionally exhibiting [8-15] pressure agyrotropy $\hat{A}^{\text {n.g. }}$. An example is provided by the distribution functions of ions flowing out into the upstream solar wind within a magnetic flux tube [11]. The generation of nongyrotropic anisotropy by a shear flow was noted in a Vlasov plasma [22,23], where its competition with secondary anisotropy-driven instabilities was discussed. A velocity shear plays an important role in the enhancement of a variety of pressure anisotropy-related plasma instabilities, such as the ion-Weibel modes in the geomagnetic tail, whose threshold is known [24] to be lowered by the presence of a velocity shear in the near-Earth plasma-sheet profile prior to a substorm expansion. In addition, in a fast solar wind [18] and in "space simulation experiments" [19] multipeaked particle distribution functions turn out to be correlated with the magnitude of the gyrotropic anisotropy of the core protons, which is generally otherwise interpreted [20] within the CGL framework.

The anisotropization mechanism, discussed, for the sake of simplicity, in this paper for ions, can obviously be extended to the generation of a nongyrotropic electron pressure tensor. The latter has been indicated as the dominant nonideal term in Ohm's law driving magnetic reconnection in low-collisionality regimes [25-27]. Nongyrotropic electron distributions have been observed in the magnetopause [12-14] next to $X$ and $O$ points in the reconnection diffusion region, which are 
known to be hyperbolic points for convection flows [28]. The generation of pressure agyrotropy near a steady reconnecting $X$ point due to the local velocity shear was noted in [29], though with an analysis different from the one presented here. Moreover, in the nonlinear stage of the current-filamentation instability arising in the presence of two opposite cold electron beams, an anisotropic pressure tensor was shown to form and to decrease the threshold of and to increase the growth rate of the reconnection instability developing on the shoulder of the magnetic structures generated by current-filamentation instability [30], which are also encountered in the presence of radially inhomogeneous beams such as in high-intensity laser-plasma interactions [31] and are measured in laboratory experiments [32].

We introduce the equations of the model in Sec. II and analyze the pressure tensor dynamics in Sec. III, where we show the role of the traceless strain in modifying the internal energy of the plasma and in generating pressure agyrotropy from an initially isotropic state. The description of the shear-induced anisotropization mechanism takes a particularly simple form when assuming invariance along the initial magnetic field, as is the case in all the examples considered in the article, and allows for a polar coordinate representation of the in-plane pressure tensor in terms of the local, instantaneous, normalized agyrotropy $\hat{A}^{\text {n.g. }}$ of the in-plane internal energy and of the angle of rotation of the principal axes of the pressure tensor (Sec. III A). Two examples of this analysis are then considered. First, the solutions of the pressure tensor equation are found by assuming an external forcing which makes the magnetic and velocity fields constant in time (Sec. IV). Second, the generation of both agyrotropic and gyrotropic anisotropy is evidenced by numerical integration of the full set of governing equations, and the numerical results are interpreted in terms of the general analysis presented in Sec. III A and in terms of the normal modes which can propagate in the system [3] (Sec. V). The results are discussed and summarized in Sec. VI.

\section{GOVERNING EQUATIONS}

We start from the two-fluid equations of a collisionless magnetized plasma obtained from the moments of the Vlasov equation [3,7,33] coupled to Maxwell's equations, where we have assumed quasineutrality and neglected the displacement current. We simplify the electron dynamics by taking $m_{e} / m_{i} \rightarrow 0$ and by neglecting the electron temperature, whereas the full ion pressure tensor, defined as $\boldsymbol{\Pi} \equiv$ $\int f_{i}(x, v, t) m_{i} \boldsymbol{v} \boldsymbol{v} d^{3} v-n m_{i} \boldsymbol{u} \boldsymbol{u}$, with $f_{i}(x, v, t)$ ion distribution function, $n$ ion density, and $\boldsymbol{u}$ ion fluid velocity, contributes to the plasma dynamics:

$$
\frac{\partial n}{\partial t}=-\nabla \cdot(n \boldsymbol{u}), \quad \frac{\partial \boldsymbol{u}}{\partial t}+\boldsymbol{u} \cdot \nabla \boldsymbol{u}=\frac{\boldsymbol{J} \times \boldsymbol{B}}{n m_{i} c}-\frac{\nabla \cdot \boldsymbol{\Pi}}{n m_{i}} .
$$

The magnetic field evolves according to the ideal Hall-MHD induction equation,

$$
\frac{\partial \boldsymbol{B}}{\partial t}=\nabla \times\left(\frac{\boldsymbol{u}-\boldsymbol{J} /(n e)}{c} \times \boldsymbol{B}\right),
$$

while the time evolution of the ion pressure tensor $\Pi$ is given by

$$
\begin{aligned}
& \frac{\partial \boldsymbol{\Pi}}{\partial t}+\nabla \cdot(\boldsymbol{u} \boldsymbol{\Pi})+(\nabla \boldsymbol{u}) \cdot \boldsymbol{\Pi}+((\nabla \boldsymbol{u}) \cdot \boldsymbol{\Pi})^{T} \\
& \quad=\Omega_{c}(\boldsymbol{\Pi} \times \boldsymbol{b}+\boldsymbol{b} \times \boldsymbol{\Pi}) .
\end{aligned}
$$

Here $\Omega_{c} \equiv q|\boldsymbol{B}| /(m c), \boldsymbol{b} \equiv \boldsymbol{B} /|\boldsymbol{B}|$, and $T$ denotes the matrix transpose. The simplifying assumption of neglecting in Eq. (3) the divergence of the ion heat flux, $Q_{i j k} \equiv\left\langle m n\left(v_{i}-\right.\right.$ $\left.\left.u_{i}\right)\left(v_{j}-u_{j}\right)\left(v_{k}-u_{k}\right)\right\rangle$, is consistent [3] with the geometrical configuration considered later in this paper, at least until very short spatial scales in the plane perpendicular to the magnetic field are nonlinearly generated. The terms in Eq. (3) can be collected according to their characteristic time scales as

$$
\frac{\partial}{\partial t} \boldsymbol{\Pi}=-\underbrace{\mathcal{L}_{\mathbf{u}}(\boldsymbol{\Pi})}_{|\nabla \mathbf{u}| \equiv \tau_{H}^{-1}}+\underbrace{\mathcal{M}_{\mathbf{u}}(\boldsymbol{\Pi})}_{\Omega_{c} \equiv \tau_{B}^{-1}},
$$

where we have introduced the linear operators $\mathcal{L}_{\mathbf{u}}\left(\Pi_{i j}\right) \equiv \partial_{k}\left(u_{k} \Pi_{i j}\right)+\Pi_{k j} \partial_{k} u_{i}+\Pi_{i k} \partial_{k} u_{j}$ and $\mathcal{M}_{\mathbf{u}}\left(\Pi_{i j}\right) \equiv$ $\Omega_{c}\left(\varepsilon_{i l m} \Pi_{l j} b_{m}+\varepsilon_{j l m} \Pi_{i l} b_{m}\right)$, which correspond to the characteristic hydrodynamic $\left(\tau_{H} \equiv|\nabla \mathbf{u}|^{-1}\right)$ and magnetic $\left(\tau_{B} \equiv \Omega_{c}^{-1}\right)$ time scales. Typical closures of $\Pi$ are obtained by identifying a small expansion parameter, e.g., a small $\tau_{B} / \tau_{H}$ leads to finite-Larmor-radius (FLR) gyrotropic corrections to CGL equations [33-38]. Here we do not assume the ratio $\tau_{B} / \tau_{H}$ to be low. The system of Eqs. (1)-(3) goes beyond the CGL-FLR approach and, differently from the latter, at perpendicular propagation allows for a consistent description of the FLR dispersive effects on the-low frequency magnetosonic branch (LFB) in agreement with the Vlasov equation and admits a high frequency branch (HFB) corresponding to an $m=2$ ion-Bernstein wave, resonant at $\omega= \pm 2 \Omega_{c}[3]$.

\section{ROLE OF THE VELOCITY STRAIN}

Defining the matrices $\mathbf{B}_{i j} \equiv \Omega_{c} \varepsilon_{i j m} b_{m}$ and $\mathbf{W}_{i j} \equiv\left(\partial_{i} u_{j}-\right.$ $\left.\partial_{j} u_{i}\right) / 2$, which describe the rotation induced by the magnetic field and by the shear flow, respectively, the strain traceless matrix $\mathbf{D}_{i j} \equiv\left(\partial_{j} u_{i}+\partial_{i} u_{j}\right) / 2+C \boldsymbol{\delta}_{i j}$, the volumetric compressibility in three-dimensional space $C \equiv-\left(\partial_{k} u_{k}\right) / 3$, and the derivative $d / d t \equiv \partial_{t}+u_{k} \partial_{k}$, Eq. (3) can be written as

$$
\frac{d}{d t} \boldsymbol{\Pi}=[\mathbf{B}+\mathbf{W}, \boldsymbol{\Pi}]-\{\mathbf{D}, \boldsymbol{\Pi}\}+5 C \boldsymbol{\Pi},
$$

where [,] denotes the commutator and $\{$,$\} the anticommutator.$ The compressibility term $C$ acts isotropically on $\Pi$, while the commutator term shows that the magnetic field $\boldsymbol{B}$ and the flow vorticity $\boldsymbol{\omega}\left(\omega_{i} \equiv \varepsilon_{i j k} W_{k j}\right)$ combine to make $\boldsymbol{\Pi}$ rotate around the axis of $\mathbf{B}+\mathbf{W}$. The perpendicular components rotate at twice the cyclotron frequency in the absence of vorticity or at twice the fluid rotation frequency in the vanishing magnetic-field limit. If the axes of $\mathbf{B}$ and $\mathbf{W}$ are aligned, the two frequencies add up if $\boldsymbol{B} \cdot \boldsymbol{\omega}>0$ and subtract if $\boldsymbol{B} \cdot \boldsymbol{\omega}<0$. The role of this asymmetry was noted in a CGL-FLR framework [39], in the evolution of the $\mathrm{KH}$ developing at the dusk and dawn flanks of planetary or cometary magnetospheres. It also intervenes in the onset of the shear-induced mechanism which drives the anisotropization that is described below. 
The traceless strain $\mathbf{D}$ can modify the internal energy of the plasma (i.e., $1 / 2$ the trace of $\Pi$ ) independently of isotropic compressions,

$$
\frac{d}{d t} \operatorname{tr}\{\boldsymbol{\Pi}\}=-2 \operatorname{tr}\{\mathbf{D} \boldsymbol{\Pi}\}+5 C \operatorname{tr}\{\boldsymbol{\Pi}\},
$$

and, through the anticommutator term of Eq.(5), can induce both gyrotropic and nongyrotropic pressure anisotropization, as can be shown by projecting the pressure tensor $\Pi$ along the rotation axis of $\mathbf{B}+\mathbf{W}$ and onto the perpendicular plane.

\section{A. Agyrotropy generation}

In the examples discussed in this article we consider a uniform initial magnetic field directed along the $z$ axis and assume that all quantities are constant along $z\left(\partial_{z}=0\right)$. In this geometry the Hall term in Eq. (2) vanishes identically [3], so that the magnetic field remains aligned along $z$, though possibly evolving in magnitude because of the threedimensional compressibility term. In this limit the dynamics of the pressure tensor $\Pi_{\perp}$ in the $x-y$ plane does not depend on the remaining components and we can thus project Eq. (3) onto this plane. In doing so, in order to keep the strain D traceless, it is convenient to adopt a two-dimensional, in-plane, compressibility $C_{\perp}$, defined as $C_{\perp} \equiv-\left(\partial_{k} u_{k}\right) / 2$ with $k=x, y$. Then, in lieu of Eq. (5), we obtain

$$
\frac{d}{d t} \boldsymbol{\Pi}_{\perp}=\left[\mathbf{B}+\mathbf{W}, \boldsymbol{\Pi}_{\perp}\right]-\left\{\mathbf{D}, \boldsymbol{\Pi}_{\perp}\right\}+4 C_{\perp} \boldsymbol{\Pi}_{\perp},
$$

where all operators can be written as $2 \times 2$ matrices and $\mathbf{B}+\mathbf{W}=\left(\Omega_{c}+\omega_{z} / 2\right) \mathbf{L}$ with $\Omega_{c}$ is the cyclotron frequency, $\omega_{z}$ the $z$ component of the vorticity, and $\mathbf{L}$ the unitary antisymmetric rotation matrix with $\mathbf{L}_{x x}=\mathbf{L}_{y y}=0$ and $\mathbf{L}_{x y}=$ $\mathbf{L}_{y x}=1$. Defining the agyrotropic part of the perpendicular pressure tensor as $\mathbf{A}^{\text {n.g. }} \equiv \boldsymbol{\Pi}_{\perp}-\operatorname{tr}\left\{\boldsymbol{\Pi}_{\perp}\right\} \mathbf{I} / 2$, from Eq. (7) we obtain

$$
\begin{aligned}
\frac{d}{d t} \mathbf{A}^{\text {n.g. }}= & {\left[\mathbf{B}+\mathbf{W}, \mathbf{A}^{\text {n.g. }}\right]-\left\{\mathbf{D}, \mathbf{A}^{\text {n.g. }}\right\}+4 C_{\perp} \mathbf{A}^{\text {n.g. }} } \\
& +\mathbf{I} \operatorname{tr}\left\{\mathbf{D} \mathbf{A}^{\text {n.g. }}\right\}-\mathbf{D} \operatorname{tr}\left\{\boldsymbol{\Pi}_{\perp}\right\} .
\end{aligned}
$$

It is convenient to define a normalized agyrotropy $\hat{A}^{\text {n.g. }}(0 \leqslant$ $\left.\hat{A}^{\text {n.g. }} \leqslant 1\right)$ which is related to the eigenvalues $\pm A^{\text {n.g. }}$ of the agyrotropic part of the perpendicular pressure tensor by

$$
\hat{A}^{\text {n.g. }} \equiv \frac{2 A^{\text {n.g. }}}{\operatorname{tr}\left\{\Pi_{\perp}\right\}} .
$$

Introducing polar coordinates according to

$$
\begin{aligned}
\Pi_{x y} & =A^{\text {n.g. }} \sin 2 \theta, & \frac{\Pi_{x x}-\Pi_{y y}}{2}=A^{\text {n.g. }} \cos 2 \theta, \\
D_{x y} & =D \sin 2 \phi, & \frac{D_{x x}-D_{y y}}{2}=D \cos 2 \phi,
\end{aligned}
$$

we can rewrite Eq. (7) as a system of three coupled scalar equations,

$$
\begin{gathered}
\frac{d}{d t} \operatorname{tr}\left\{\boldsymbol{\Pi}_{\perp}\right\}=-4 A^{\text {n.g. }} D \cos [2(\theta-\phi)]+4 C \operatorname{tr}\left\{\Pi_{\perp}\right\}, \\
\frac{d A^{\text {n.g. }}}{d t}=-D \operatorname{tr}\left\{\Pi_{\perp}\right\} \cos [2(\theta-\phi)]+4 C A^{\text {n.g. }}, \\
2 \frac{d \theta}{d t}=-\left(2 \Omega_{c}+\omega_{z}\right)+D \frac{\operatorname{tr}\left\{\Pi_{\perp}\right\}}{A^{\text {n.g. }}} \sin [2(\theta-\phi)] .
\end{gathered}
$$

Only the anticommutator and the compression term in Eq. (7) contribute to the right-hand side (r.h.s.) of Eqs. (12) and (13) and to the second r.h.s. term in Eq. (14), whereas the commutator is responsible for the first r.h.s. term in Eq. (14). If $A^{\text {n.g. }}=0$ at $t=0$ (initial in-plane pressure isotropy), the angle $\theta(\boldsymbol{x}, 0)$ is undefined but the relative phase between $\theta(\boldsymbol{x}, 0)$ and $\phi(\boldsymbol{x}, 0)$ can be determined by direct comparison of the components of Eq. (7) written in polar and in Cartesian coordinates, which yields $\theta(\boldsymbol{x}, 0)=\phi(\boldsymbol{x}, 0)+\pi / 2$.

Equation (14) shows that the rotation frequency of the agyrotropic components of the perpendicular pressure tensor is modified by the velocity strain through a term that depends on $\sin [2(\theta-\phi)]$. Even when the strain contribution in Eq. (14) remains smaller than the $2 \Omega_{c}+\omega_{z}$ term, i.e., when $d \theta / d t$ never vanishes and there is no inversion of the rotation, the strain term can lead to a nonzero time average of the agyrotropic pressure tensor components in Eq. (11). For example, it is easily seen that if we take $\phi$ and the ratio $D \operatorname{tr}\left\{\boldsymbol{\Pi}_{\perp}\right\} / A^{\text {n.g. }}$ to be nearly constant over a rotation period, then $\langle\sin [2(\theta-\phi)]\rangle \neq 0$, while $\langle\cos [2(\theta-\phi)]\rangle=0$. Here \langle\rangle denotes the time average over a rotation period. This indicates that a slowly varying velocity strain induces a net agyrotropy in the in-plane pressure with an angular shift of $\pi / 2$.

From Eqs. (12) and (13) we obtain the evolution of the normalized agyrotropy,

$$
\frac{d \hat{A}^{\text {n.g. }}}{d t}=2 D\left[\left(\hat{A}^{\text {n.g. }}\right)^{2}-1\right] \cos [2(\theta-\phi)],
$$

which is independent of the compressibility term. Inspection of Eqs. (13) and (14) shows that both $A^{\text {n.g }}$ and $\hat{A}^{\text {n.g. increase }}$ when the principal axes of $\boldsymbol{\Pi}_{\perp}$ and $\mathbf{D}$ are dephased by an angle comprised between $\pi / 4$ and $\pi / 2$. The maximum rate of increase is obtained when the minor axis of the perpendicular pressure tensor is aligned with the major axis of the traceless strain (and vice versa).

The above equations must be supplemented by the equations for the plasma fluid velocity $\boldsymbol{u}$ in the $x-y$ plane and for the $z$ component of the magnetic field $\boldsymbol{B}$ as given in Eqs. (1) and (2).

\section{FORCED SOLUTIONS}

In order to obtain explicit solutions to the system of equations describing the growth of agyrotropy derived in Sec. III A, as the first step we consider a model plasma configuration with an incompressible shear flow $u_{y}^{0}(x)$ constant in time (energy is thus constantly injected into the system) in the presence of a uniform and constant magnetic field along the $z$ axis. In this model configuration the velocity strain and the vorticity have the same magnitude. Since $\mathbf{B}$ is uniform in space, the axes of $\mathbf{B}$ and $\mathbf{W}$ are aligned along $z$, and $\mathbf{D}$ has no $z$ components, the conditions are as described in Sec. III A with the additional simplification that Eq. (7) reduces to a linear system of constant coefficient equations. It is thus convenient to follow an eigenvalue analysis so as to identify oscillatory and purely growing regimes. We find three eigenvalues, $\gamma_{0}=0$, which corresponds to a stationary agyrotropic configuration with

$$
\frac{\Pi_{y y}^{\gamma_{0}}}{\Pi_{x x}^{\gamma_{0}}}=\frac{\Omega^{\prime}(x)}{\Omega_{c}}, \quad \Pi_{x y}^{\gamma_{0}}=0,
$$




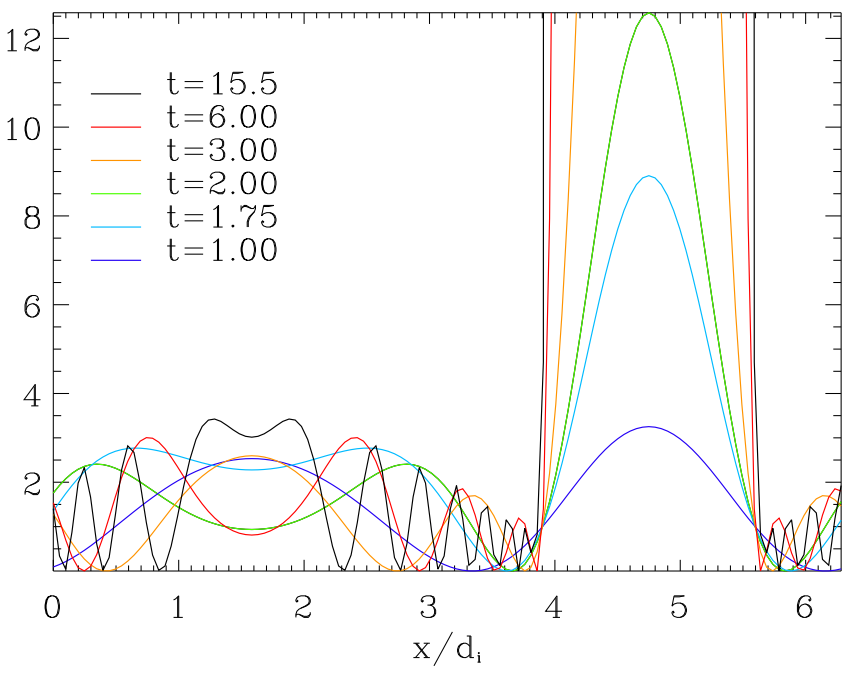

FIG. 1. Evolution of $\Pi_{y y}(x, t)$ for $\boldsymbol{B}=B_{0} \boldsymbol{e}_{z}$ and constant $\boldsymbol{u}=$ $\left(0, V_{0} \cos \left(x / d_{i}\right), 0\right), \Omega_{c} \tau_{H}=1$, and $V_{0}=-1.5 c_{A}$. Both the exponential growth $\left(\Omega^{\prime}(x)>0\right)$ and the spatial filamentation of the oscillating solutions $\left(\Omega^{\prime}(x)<0\right)$ are visible.
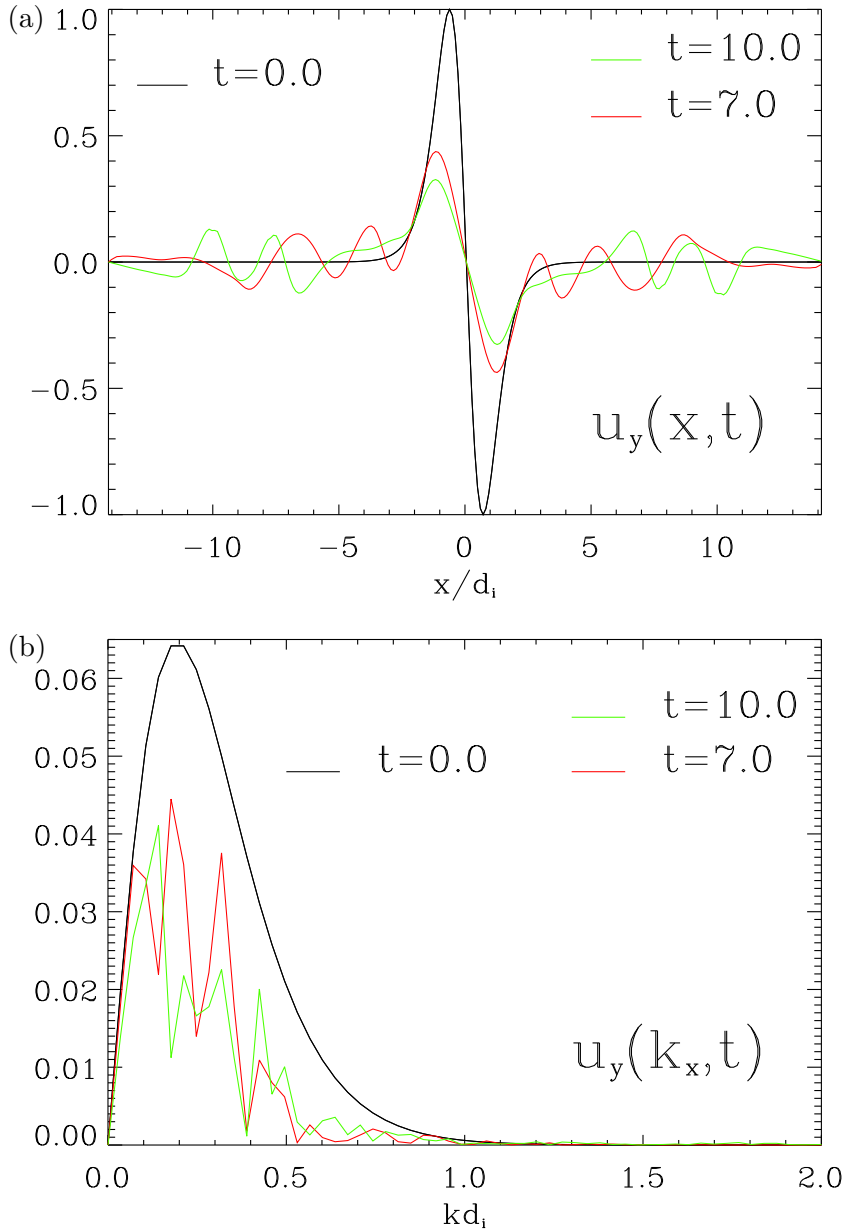

FIG. 2. Profiles of (a) $u_{y}(x, t)$ and (b) its Fourier spectrum, for $c_{H}=c_{\perp}=c_{A}=1$; times are in units of $\tau_{H}=\tau_{B}$. and two oscillatory or growing modes $\gamma_{ \pm}= \pm 2 i \sqrt{\Omega_{c} \Omega^{\prime}(x)}$ with "polarizations"

$$
\frac{\Pi_{y y}^{\gamma_{ \pm}}}{\Pi_{x x}^{\gamma_{ \pm}}}=-\frac{\Omega^{\prime}(x)}{\Omega_{c}}, \quad \frac{\Pi_{x y}^{\gamma_{ \pm}}}{\Pi_{x x}^{\gamma_{ \pm}}}= \pm i \sqrt{\frac{\Omega^{\prime}(x)}{\Omega_{c}}} .
$$

Here $\Omega^{\prime}(x) \equiv \Omega_{c}+\partial_{x} u_{y}^{0}(x)$. Provided $\Omega^{\prime}(x)>0$, the $\gamma_{0}$ mode can describe an equilibrium solution of Eq. (5) (in agreement with the self-consistent equilibria discussed in [40]), $\Pi_{y y} / \Pi_{x x}=\Omega^{\prime}(x) / \Omega_{c}$ and $\Pi_{x y}=0$. The $\gamma_{ \pm}$modes represent either oscillations or growing and damped modes, depending on the sign of $\Omega^{\prime}(x)$. For $\Omega^{\prime}(x)>0$ the perpendicular pressure tensor components of an initial isotropic state with $\Pi_{x x}(x, 0)=$ $\Pi_{y y}(x, 0)=P_{\perp}(x)$ oscillate in time around a mean value given by

$$
\left\langle\Pi_{y y}(x, t)\right\rangle=\frac{\Omega^{\prime}(x)}{\Omega_{c}}\left\langle\Pi_{x x}(x, t)\right\rangle=\left(\Omega^{\prime}(x)+\Omega_{c}\right) \frac{P_{\perp}(x)}{2 \Omega_{c}}
$$

and $\left\langle\Pi_{x y}(x, t)\right\rangle=0$, which is consistent with the comment on the rotation averages given following Eq. (14) in Sec. III A. The amplitude of the oscillations of $\Pi_{y y}(x, t)$ is given by $\partial_{x} u_{y}^{0}(x) P_{\perp}(x, 0) /\left(4 \Omega_{c}\right)$. In Fig. 1 the profile of $\Pi_{y y}$ is shown at different times, for an initial pressure tensor $\Pi_{i j}=\delta_{i j}, B_{z}^{0}=1$ and $u_{y}^{0}=V_{0} \cos (k x)$ with $V_{0}=-1.5$ and $k=1$. An important
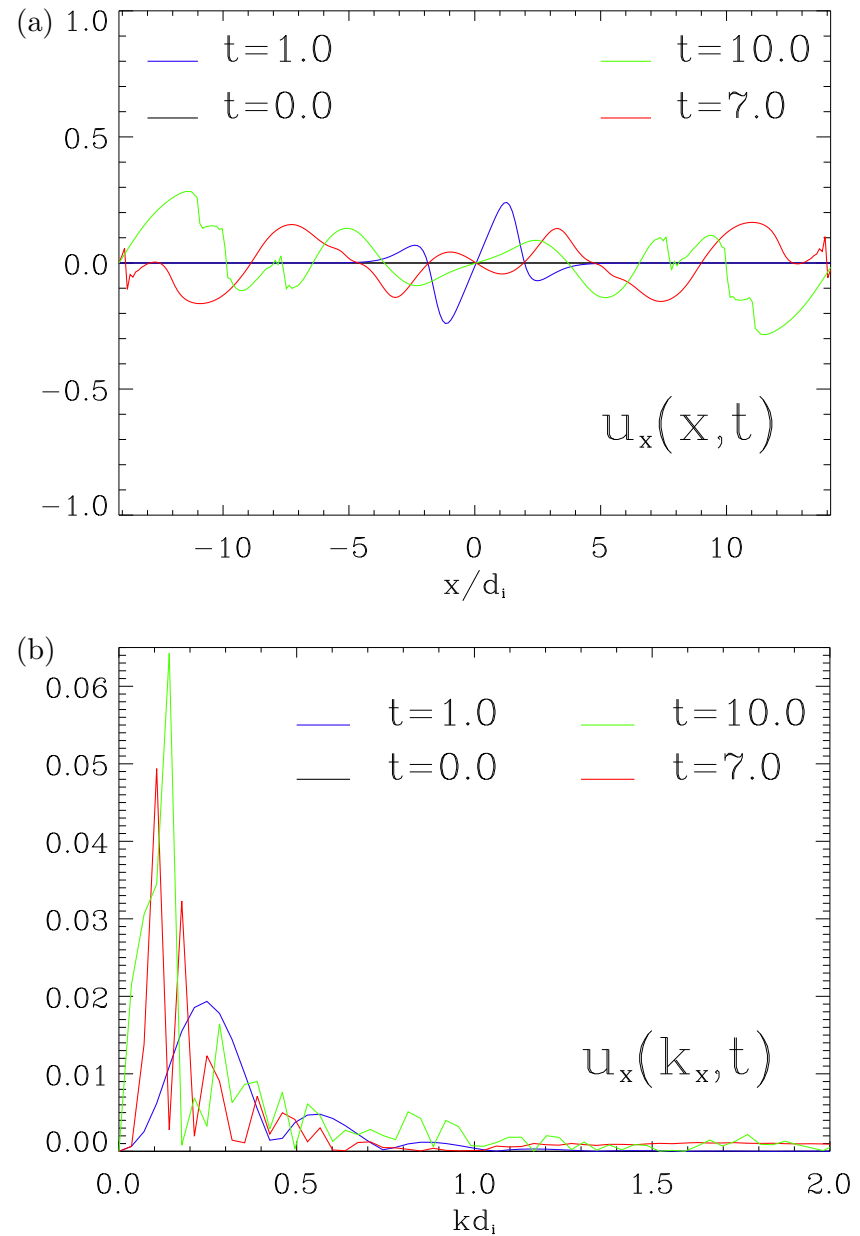

FIG. 3. Profiles of (a) $u_{x}(x, t)$ and (b) its Fourier spectrum, for $c_{H}=c_{\perp}=c_{A}=1$; times are in units of $\tau_{H}=\tau_{B}$. 
feature caused by the spatial inhomogeneity of the shear flow is the strongly inhomogeneous growth of the components of the pressure tensor as regions where the evolution is oscillatory alternate, depending on the local sign of $\Omega^{\prime} \Omega_{c}$, with regions of exponential growth occurring over a time scale $\tau_{H}=\left(k V_{0}\right)^{-1}$. This gives rise to a spatially filamented pressure tensor. In this example, when the instability condition $\Omega^{\prime}(x)<0$ is satisfied, it is easy to verify both from Eq. (17) and from Eqs. (12) and (13) that the trace of $\Pi_{\perp}$ and the non-normalized agyrotropy $A^{\text {n.g. }}$ exponentially grow in time at a rate $\gamma_{+}$, while

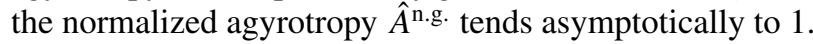

\section{SELF-CONSISTENT NUMERICAL SOLUTIONS}

In this section we consider the time evolution of the pressure tensor in the self-consistent case, in which the flow and the electromagnetic fields evolve according to Eqs. (1)-(3): here the anisotropization of the pressure tensor caused by the presence of an initially imposed shear flow is limited by the action of the pressure tensor on the plasma flow, which reduces

(a)

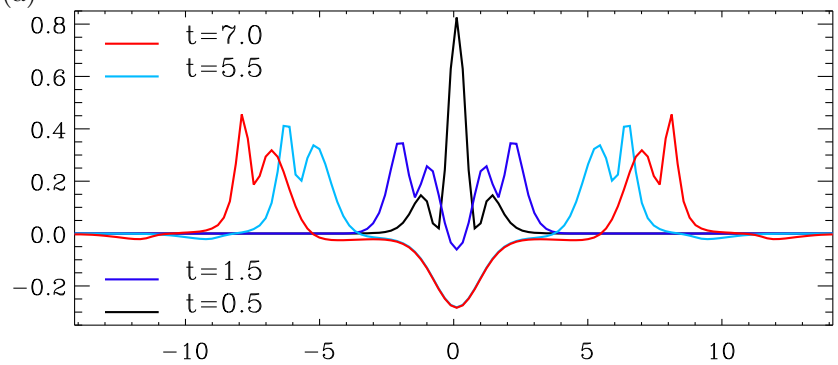

(b)
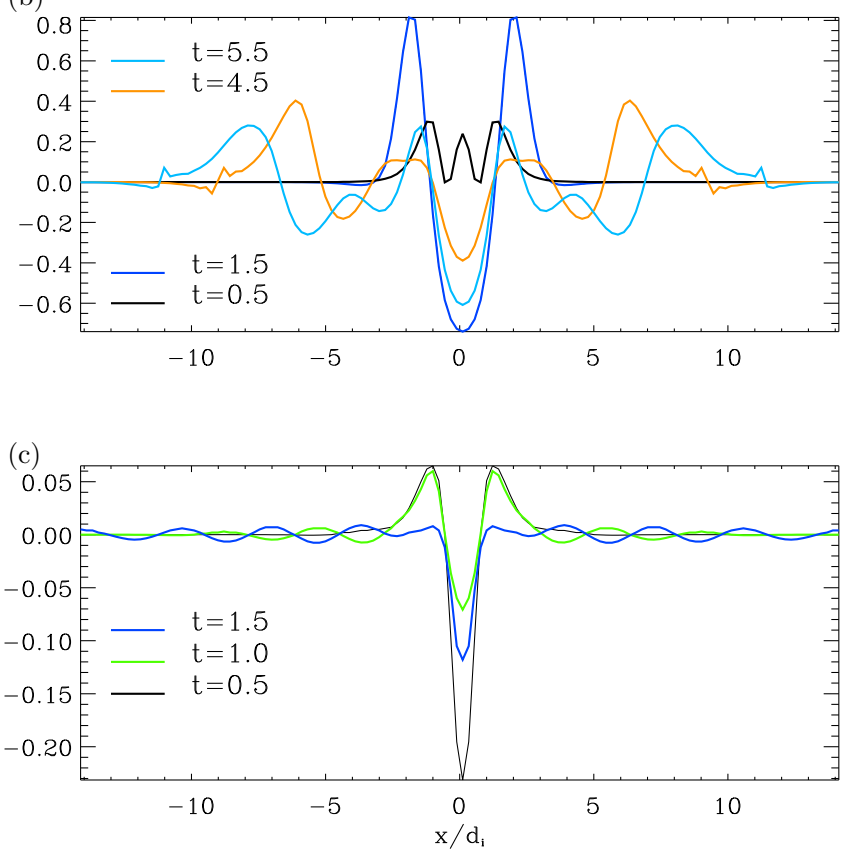

its shear, and by the excitation of nonlinear magnetoelastic perturbations, which tend to redistribute the shear of the velocity flow. This system conserves the total energy

$$
E^{\mathrm{tot}}=\int d \boldsymbol{x}^{3}\left(\frac{n m_{i} u^{2}}{2}+\frac{B^{2}}{8 \pi}+\frac{\operatorname{tr}\{\boldsymbol{\Pi}\}}{2}\right)
$$

and depends on three dimensionless parameters, $\tau_{H} / \tau_{B}=$ $\left(c_{A} / c_{H}\right)\left(L_{H} / d_{i}\right),\left(c_{A} / c_{H}\right)^{2}$, and $\left(c_{\perp} / c_{H}\right)^{2}$, with $L_{H}$ the scale length of the configuration, $c_{A}$ the Alfvèn velocity, $c_{H}=$ $L_{H} / \tau_{H}$ a measure of the flow velocity, $d_{i} \equiv c_{A} / \Omega_{c}$ the ion skin depth, and $c_{\perp}^{2} \equiv P_{\perp} /\left(n m_{i}\right)=c_{s}^{2} / 2$, with $c_{s}$, the "sound" velocity evaluated with respect to the initial ion pressure, assumed isotropic in the plane perpendicular to $\boldsymbol{B}$ [3]. Only two parameters, $\tau_{H} / \tau_{B}$ and $\left(c_{\perp} / c_{A}\right)^{2}$, rule the linear dynamics.

The nonlinear self-consistent case has been integrated numerically starting from an isotropic initial condition with homogeneous density, $\boldsymbol{B}=B_{0} \boldsymbol{e}_{z}$ and $\boldsymbol{u}=u_{y}^{0}(x) \mathbf{e}_{y}$, varying the value of the ratios of the three dimensionless parameters. In Figs. 2-7 we consider the case with $u_{y}^{0}(x)=$

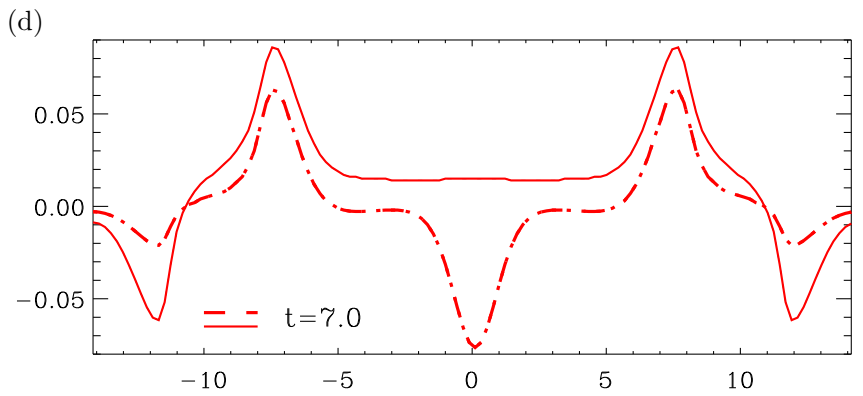

(e)

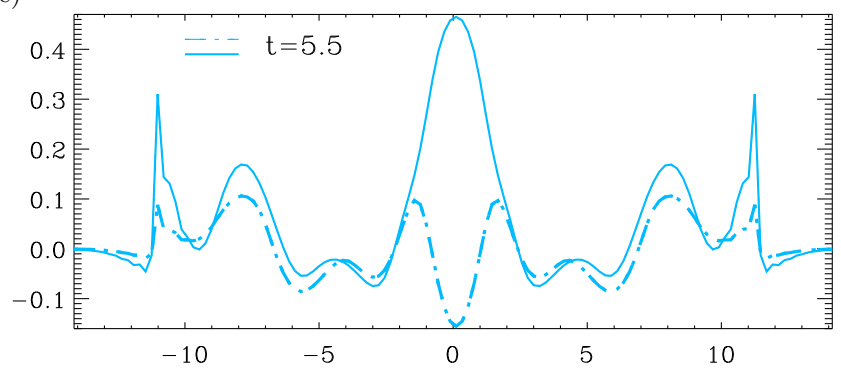

(f)

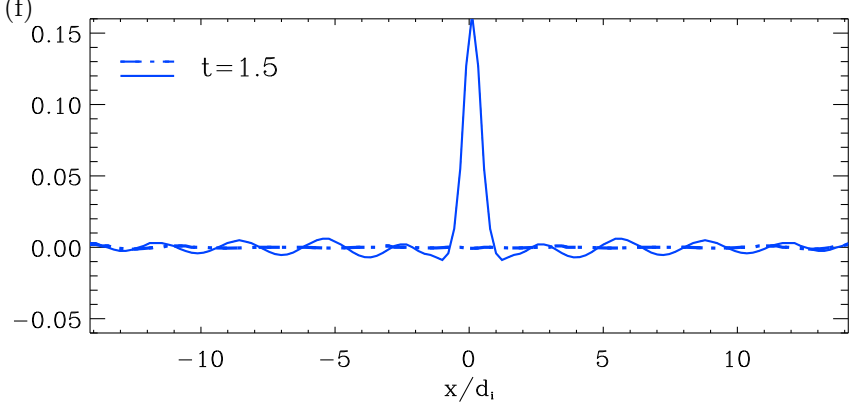

FIG. 4. Spatial profiles of the local difference from the initial value for $(\mathrm{a}-\mathrm{c}) \delta \Pi_{y y}(x, t) \equiv \Pi_{y y}(x, t)-\Pi_{y y}(x, 0),(\mathrm{d}-\mathrm{f}) \delta \Pi_{x x}(x, t) \equiv$ $\Pi_{x x}(x, t)-\Pi_{x x}(x, 0)$ (solid lines) and $\delta \Pi_{z z}(x, t) \equiv \Pi_{z z}(x, t)-\Pi_{z z}(x, 0)$ (dash-dotted lines). The initial pressures are uniform and isotropic $\left(\Pi_{i j}^{0}=\delta_{i j}\right)$ with $c_{\perp} / c_{H}=1$ and $L_{H}=d_{i}$; times are in units of $\tau_{H}$. From top to bottom, the values $\tau_{H} / \tau_{B}=c_{A} / c_{H}=0.1,1$, and 10 correspond to the pairs of frames in each row, i.e., to (a) and (d), to (b) and (e), and to (c) and (f), respectively. In (c) and (f) the magnetosonic waves leave the box earlier and their amplitude decreases because of the increased value of $B_{0}$ (cf. LFB polarization). 
$V_{0} \tanh \left(x / d_{i}\right) / \cosh ^{2}\left(x / d_{i}\right)$ and $\tau_{H} / \tau_{B}=c_{A} / c_{H}=c_{\perp} / c_{H}=$ 1. Note that, though its characteristic scale length is chosen of the order $d_{i}$, the initial Fourier spectrum peaks around $k d_{i} \lesssim 1$ (Fig. 2).

The results obtained show a wave-like behavior of the initial spatially localized velocity that can be qualitatively accounted for by interpreting the shear velocity $u_{y}^{0}(x)$ as an initial perturbation described as a superposition of magnetoelastic modes. These oscillatory modes, with perturbed velocities in the $x-y$ plane, propagating along the $x$ axis are obtained by solving the linearized Eqs. (1), (2), and (7) around a steady spatially homogeneous equilibrium with a uniform magnetic field in the $z$ direction. As detailed in Ref. [3] this system of linearized equations describes two oscillation branches: a low-frequency branch (LFB), which is an extension of the standard magnetosonic mode, and a high-frequency branch (HFB), which is induced by the dynamics of the pressure tensor. To leading order in $k d_{i} \ll 1$, the LFB and HFB have dispersion relations

$$
\omega_{l}^{2} \sim k^{2}\left(c_{A}^{2}+2 c_{\perp}^{2}\right), \quad \omega_{h}^{2} \sim 4 \Omega^{2}+2 k^{2} c_{\perp}^{2}
$$

and polarization vector components $\{1, i o(\varepsilon)\}_{l}$ and $\{1,-i\}_{h}$ in the $\left\{u_{x}, u_{y}\right\}$ basis, with $\varepsilon \sim k c_{\perp}^{2} /\left(2 \Omega_{c} v_{g, l}\right)$ and $v_{g, l} \sim\left(c_{A}^{2}+\right.$ $\left.2 c_{\perp}^{2}\right)^{1 / 2}$ group velocity of the LFB. Then, ordering $c_{\perp} \sim c_{A}$, the LFB polarization vector results, $\left\{1, i o\left(k d_{i}\right)\right\}_{l}$. This implies that the chosen initial perturbation can be interpreted as a superposition of the two branches with equal and opposite $u_{x}$ amplitudes and that the time evolution of $u_{y}^{0}(x)$ is mainly determined by that of the HFB, whose group velocity for $k d_{i} \ll 1, v_{g, h} \sim\left(k d_{i}\right) c_{\perp}^{2} / c_{A}$, decreases linearly with $B_{0}$ and vanishes for $k \rightarrow 0$. On the contrary, both branches contribute to the evolution of $u_{x}(x)$, where the initial cancellation is removed as time evolves with the LFB component propagating outwards and the HFB essentially mirroring (Fig. 3) the behavior of $u_{y}$ displayed in Fig. 2. This is consistent with the results of the numerical integration and explains why the normalized pressure agyrotropy $\hat{A}^{\text {n.g. }}$, which in our geometry is mainly related to the spatial inhomogeneity of $u_{y}^{0}(x)$, tends to remain in the original position and not to be carried away at the Alfvènic group velocity of the LFB, at least until small spatial scales are formed, which are instead transported away efficiently by the HFB. This localization around $x \simeq 0$ is evident in the time evolution of the profile along $x$ of all the components of $\Pi$, as shown in Fig. 4 for different values of the characteristic parameters. The fast local anisotropization of the $\Pi_{y y}$ pressure components next to $x \simeq 0$, consistent with the analysis presented in Sec. III A and Sec. IV, as well as its relative persistence in time, is shown in Fig. 5 for the cases corresponding to Figs. 4(a) and 4(c) and in Fig. 6(b) for the case in Fig. 4(b).

Considering now more specifically the region near $x \simeq$ 0, Fig. 7(a) shows, for $\tau_{H} / \tau_{B}=1$, the generation of the initial agyrotropy over a time scale $\sim L_{H} / c_{H}$, in agreement with Eqs. (8) and (13), followed by oscillations at $\sim 2 \Omega_{c}$, consistent with Eq. (14), of the agyrotropic components of the pressure tensor around the mean value $\hat{A}^{\text {n.g. }} \simeq-0.45$ over several $\left(k c_{A}\right)^{-1}$ times. This indicates that the local agyrotropic anisotropy is long-lived in comparison to the characteristic dynamical time scales. In fact, in the case considered, only a
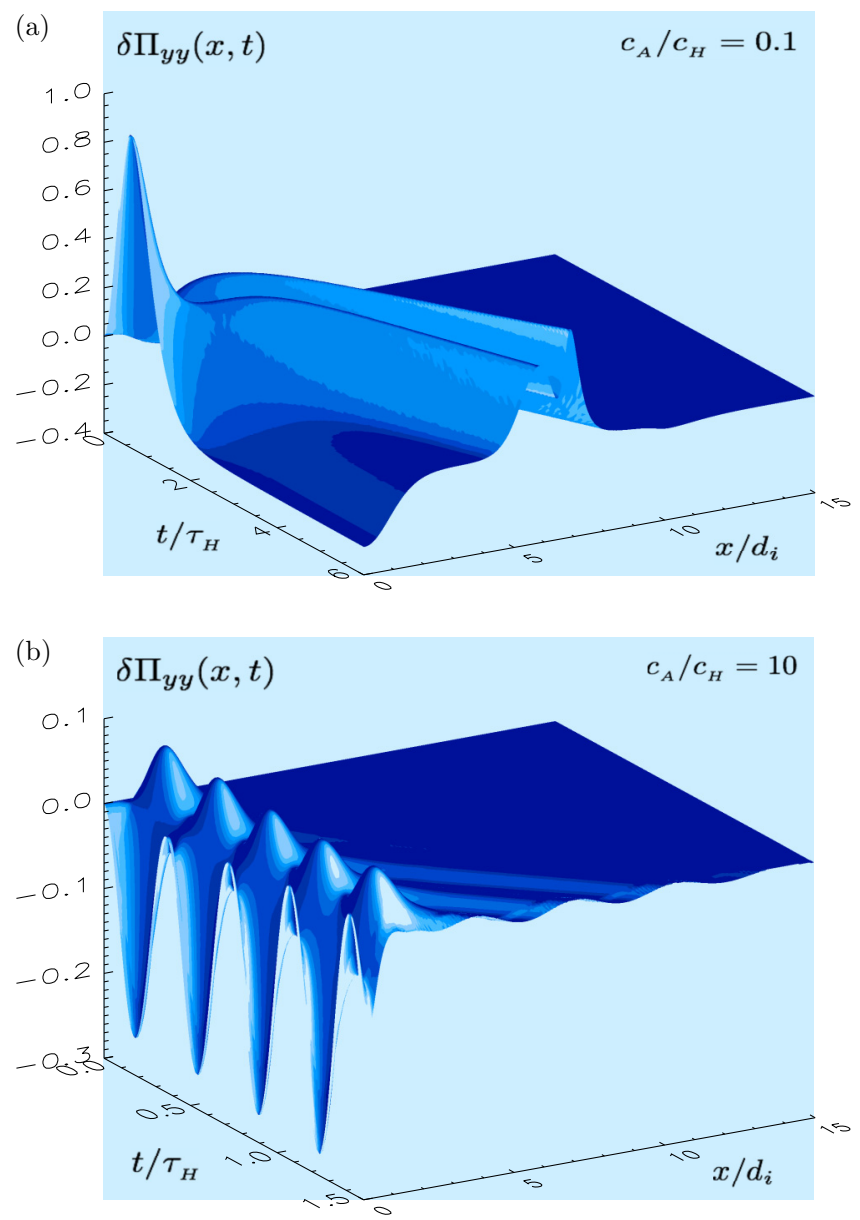

FIG. 5. Surface representation of the profiles of $\delta \Pi_{y y}(x, t)$ with respect to space (width), for $x \geqslant 0$, and time (depth), for (a) $0 \leqslant$ $t / \tau_{H} \leqslant 6$ and (b) $0 \leqslant t / \tau_{H} \leqslant 1.5$. Parameters are the same as in Figs. 4(a) and 4(c), with $c_{\perp} / c_{H}=1, L_{H}=d_{i}$, and $\tau_{H} / \tau_{B}=c_{A} / c_{H}=$ 0.1 and 10 , corresponding to (a) and (b), respectively.

fraction $\left(\lesssim k d_{i}\right.$ ) of the initial perturbation $u_{y}^{0}(x)$ is redistributed by the magnetosonic branch on the characteristic Alfvén time of the configuration, while the HFB takes a time $d_{i} / v_{g, h} \sim$ $c_{A} /\left(k c_{\perp}^{2}\right) \gg d_{i} / c_{A}=\tau_{B}$ to displace the initial velocity profile by a distance equal to its characteristic size, $d_{i}$. The oscillations of $\hat{A}^{\text {n.g. }}$ are related by Eqs. (9) and (10) to the oscillations of $\Pi_{x x}$ and $\Pi_{y y}$, shown in Figs. 6(a) and 6(b) for the same parameters, and also visible as spatial oscillations in the corresponding profiles along $x$, shown in Figs. 4(b) and 4(e) at some specific times.

For longer times the interplay between the filamentation shown in Fig. 1 and the propagation of disturbances of the pressure tensor result in the formation of fine-scale spatial structures. An example of early formation of such small-scale structures, corresponding to the steepening of the propagating perturbations, is visible in Figs. 4(b) and 4(e) next to $x \simeq$ $10.5 d_{i}$, at about $t \simeq 5.5 \tau_{H}$.

Finally, the numerical results show that, in addition to the agyrotropic anisotropy, a gyrotropic anisotropy is also generated by the initial shear velocity $u_{y}^{0}$. This can be understood within the magnetoelastic wave description, by noting that compressible fluctuations of $u_{x}$ naturally develop from the 

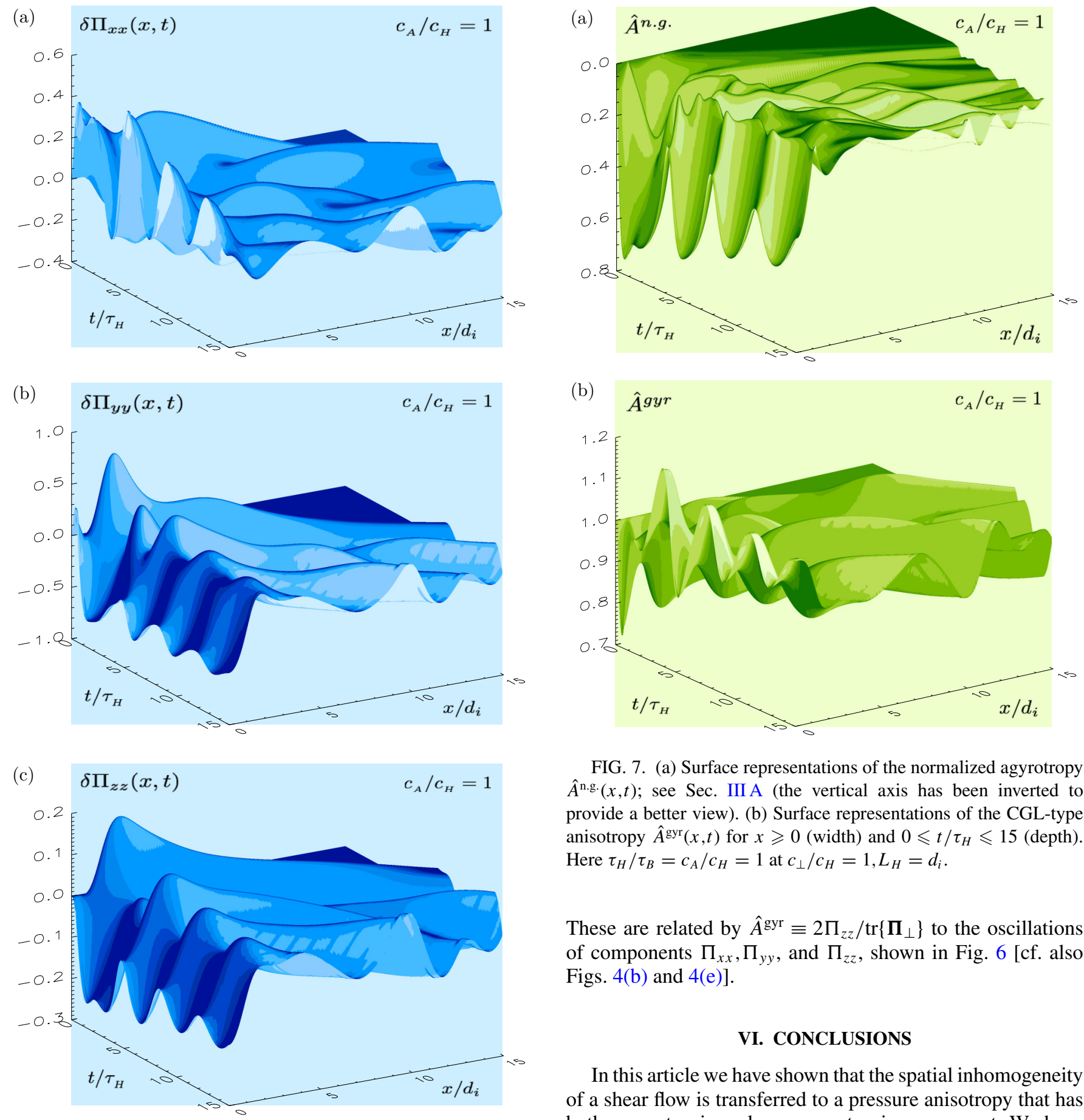

FIG. 7. (a) Surface representations of the normalized agyrotropy $\hat{A}^{\text {n.g. }}(x, t)$; see Sec. III A (the vertical axis has been inverted to provide a better view). (b) Surface representations of the CGL-type anisotropy $\hat{A}^{\text {gyr }}(x, t)$ for $x \geqslant 0$ (width) and $0 \leqslant t / \tau_{H} \leqslant 15$ (depth). Here $\tau_{H} / \tau_{B}=c_{A} / c_{H}=1$ at $c_{\perp} / c_{H}=1, L_{H}=d_{i}$.

These are related by $\hat{A}^{\mathrm{gyr}} \equiv 2 \Pi_{z z} / \operatorname{tr}\left\{\boldsymbol{\Pi}_{\perp}\right\}$ to the oscillations of components $\Pi_{x x}, \Pi_{y y}$, and $\Pi_{z z}$, shown in Fig. 6 [cf. also Figs. 4(b) and 4(e)].

\section{CONCLUSIONS}

In this article we have shown that the spatial inhomogeneity of a shear flow is transferred to a pressure anisotropy that has both a gyrotropic and a nongyrotropic component. We have investigated this process both analytically and numerically.

A consequence of this analysis that is directly relevant to of $\delta \Pi_{y y}(x, t)$, and (c) of $\delta \Pi_{z z}(x, t)$ with respect to space (width), for $x \geqslant 0$, and time (depth), for $0 \leqslant t / \tau_{H} \leqslant 15$. Parameters are the same as in Figs. 4(b) and 4(e), with $c_{\perp} / c_{H}=1, L_{H}=d_{i}$, and $\tau_{H} / \tau_{B}=$ $c_{A} / c_{H}=1$.

initially incompressible velocity profile. These induce, for both the LFB and the HFB, isothermal fluctuations of the parallel pressure [3] consistent with the magnetosonic polarization $\delta \Pi_{z z} / \Pi_{z z}^{0}=\delta B_{z} / B_{z}^{0}$ (not shown here). In Fig. 7(b) oscillations at $\sim 2 \Omega_{c}$ near $x \simeq 0$ of the gyrotropic anisotropy around a mean value of $\hat{A}^{\mathrm{gyr}} \simeq 1.05$ are shown for $\tau_{A} / \tau_{B}=1$. kinetic plasma simulations is the recognition of the need to start from an initial anisotropic distribution function in order to initialize these simulations correctly in the presence of a velocity shear [41]. In fact isotropic "MHD-type" equilibria cease to be equilibria in the presence of a stationary shear flow where nongyrotropic configurations [38,40] are instead required. This can affect the onset and development of anisotropy-driven or shear-driven instabilities, such as the KH. In fact, while the anisotropization mechanism occurs on the $\tau_{H}$ scale, the anisotropization induced by a velocity shear with a spectral distribution at $k d_{i} \lesssim 1$ is stable over a time $\sim c_{A} /\left(k c_{\perp}^{2}\right)$. On 
the other hand, the $\mathrm{KH}$ instability linear growth rate is on a $\tau_{H}$ time and so is that of the ion-Weibel mode [24] at $k d_{i} \ll 1$, while that of the "fluid" mirror instability [42] is $\sim\left(k c_{A}\right)^{-1}$. This has a direct implication for turbulence, where small-scale spatial inhomogeneities are naturally developed during the direct cascade. Since non-negligible discrepancies with respect to the CGL closure become important when $\tau_{H} \Omega_{c} \sim 1$, for $c_{H} \sim c_{A}$ (Alfvènic turbulence) pressure anisotropies in the plane perpendicular to the magnetic field can be expected when velocity inhomogeneities are generated at a scale $L_{H} \sim d_{i}$, apparently in agreement with the temperature anisotropization observed in Refs. [9] together with the development of current and vorticity layers of thickness $\sim d_{i}$. The resulting nongyrotropic state can be maintained due to the competition, noted in Refs. [22] and [23], between an external forcing ensuring the maintenance of the shear flow (e.g., turbulent convection) and secondary instabilities feeding on the pressure anisotropies.

Note added in proof. Recently an article was published [43] that presents numerical PIC-Vlasov-hybrid simulations of two-dimensional turbulence that appear to support the analysis that we have presented.

\section{ACKNOWLEDGMENTS}

The authors acknowledge useful discussions with S. S. Cerri (IPP-Garching), T. Passot and P. L. Sulem (Obs. de la Côte d'Azur), and A. Tenerani (UCLA). Part of this work was funded by FR-FCM Grants No. 1MHD.FR.12.05 and No. 1IPH.FR.13.22.
[1] S. P. Talwar, Phys. Fluids 8, 1295 (1965); N. D’Angelo, ibid. 8, 1748 (1965).

[2] G. F. Chew, M. L. Goldberger, and F. E. Low, Proc. R. Soc. London A 236, 112 (1956).

[3] D. Del Sarto, F. Pegoraro, and A. Tenerani, arXiv:1509.04938.

[4] J. Werne and D. C. Fritts, Phys. Chem. Earth B 26, 263 (2001).

[5] L. Marié and F. Daviaud, Phys. Fluids 16, 457 (2004).

[6] A. A. Schekochihin, S. C. Cowley, R. M. Kulsrud, M. S. Rosin, and T. Heinemann, Phys. Rev. Lett. 100, 081301 (2008).

[7] A. N. Kaufman, Phys. Fluids 3, 610 (1960).

[8] L. Galeotti and F. Califano, Phys. Rev. Lett. 95, 015002 (2005).

[9] S. Servidio, F. Valentini, F. Califano, and P. Veltri, Phys. Rev. Lett. 108, 045001 (2012); D. Perrone, F. Valentini, S. Servidio, S. Dalena, and P. Veltri, Astrophys. J. Lett. 762, 99 (2013).

[10] H. F. Astudillo, S. Livi, E. Marsch, and H. Rosenbauer, J. Geophys. Res.-Space 101, 24423 (1996).

[11] A. Posner, M. W. Liemohn, and T. H. Zurbuchen, Geophys. Res. Lett. 30, 1346 (2003).

[12] J. Scudder and W. Daughton, J. Geophys. Res.-Space 113, A06222 (2008).

[13] J. D. Scudder, R. D. Holdaway, W. S. Daughton, H. Karimabadi, V. Roytershteyn, C. T. Russell, and J. Y. Lopez, Phys. Rev. Lett. 108, 225005 (2012).

[14] J. D. Scudder, H. Karimabadi, W. Daughton, and V. Roytershteyn, Phys. Plasmas 22, 101204 (2015).

[15] J. He, L. Wang, C. Tu, E. Marsch, and Q. Zong, Astrophys. J. Lett. 800, L31 (2015).

[16] E. Marsch, X.-Z. Ao, and C.-Y. Tu, J. Geophys. Res.-Space 109, A04120 (2004).

[17] B. Zieger, A. Retinò, R. Nakamura, W. Baumjohann, A. Vaivads, and Y. Khotyaintsev, Geophys. Res. Lett. 38, L22103 (2011).

[18] C.-Y. Tu, E. Marsch, and Z.-R. Qin, J. Geophys. Res.-Space 109, A05101 (2004); L. Matteini, P. Hellinger, B. E. Goldstein, S. Landi, M. Velli, and M. Neugebauer, ibid. 118, 2771 (2013).

[19] E. E. Scime, P. A. Keiter, M. M. Balkey, R. F. Bolvin, J. L. Kline, M. Blackburn, and S. P. Gary, Phys. Plasmas 7, 2157 (2000).

[20] L. Matteini, P. Hellinger, S. Landi, P. M. Trávniček, and M. Velli, Space Sci. Rev. 172, 373 (2011).

[21] N. Aunai, G. Belmont, and R. Smets, J. Geophys. Res.-Space 116, A09232 (2011); N. Aunai, A. Retinò, G. Belmont, R. Smets, B. Lavraud, and A. Vaivads, Ann. Geophys. 29, 1571 (2011).
[22] F. D. Kahn, J. Fluid Mech. 14, 321 (1962); 19, 210 (1964).

[23] N. W. Albright, Phys. Fluids 13, 2728 (1970).

[24] P. H. Yoon and A. T. Y. Lui, J. Geophys. Res.-Space 101, 4899 (1996).

[25] H.-J. Cai and L. C. Lee, Phys. Plasmas 4, 509 (1997).

[26] M. Hesse and D. Winske, Geophys. Res. Lett. 99, 11177 (1994); L. Yin, D. Winske, S. P. Gary, and J. Birn, ibid. 106, 10761 (2001); L. Yin and D. Winske, Phys. Plasmas 10, 1595 (2003).

[27] H. Karimabadi, W. Daughton, and K. B. Quest, Geophys. Res. Lett. 31, L18801 (2004).

[28] D. Del Sarto, C. Marchetto, F. Pegoraro, and F. Califano, Plasma Phys. Control. Fusion 53, 035008 (2011).

[29] J. U. Brackbill, Phys. Plasmas 18, 032309 (2011).

[30] F. Califano, N. Attico, F. Pegoraro, G. Bertin, and S. V. Bulanov, Phys. Rev. Lett. 86, 5293 (2001).

[31] F. Califano, D. Del Sarto, and F. Pegoraro, Phys. Rev. Lett. 96, 105008 (2006).

[32] M. Borghesi, A. J. Mackinnon, R. Gaillard, O. Willi, A. Pukhov, and J. Meyer-ter-Vehn, Phys. Rev. Lett. 80, 5137 (1998); K. Quinn, L. Romagnani, B. Ramakrishna, G. Sarri, M. E. Dieckmann, P. A. Wilson, J. Fuchs, L. Lancia, A. Pipahl, T. Toncian, O. Willi, R. J. Clarke, M. Notley, A. Macchi, and M. Borghesi, ibid. 108, 135001 (2012); W. Fox, G. Fiksel, A. Bhattacharjee, P. Y. Chang, K. Germaschewski, S. X. Hu, and P. M. Nilson, ibid. 111, 225002 (2013).

[33] A. Macmahon, Phys. Fluids 8, 1840 (1965).

[34] B. W. Thompson, Rep. Prog. Phys. 24, 363 (1961); K. V. Roberts and J. B. Taylor, Phys. Rev. Lett. 8, 197 (1962).

[35] N. Yajima, Prog. Theor. Phys. 36, 1 (1966).

[36] M. Khanna and R. Rajaram, J. Plasma Phys. 28, 459 (1982).

[37] A. I. Smolyakov, Can. J. Phys. 76, 321 (1998).

[38] S. S. Cerri, P. Henri, F. Califano, D. Del Sarto, M. Faganello, and F. Pegoraro, Phys. Plasmas 20, 112112 (2013).

[39] J. D. Huba, Geophys. Res. Lett. 23, 2907 (1996).

[40] S. S. Cerri, F. Pegoraro, F. Califano, D. Del Sarto, and F. Jenko, Phys. Plasmas 21, 112109 (2014).

[41] G. Belmont, N. Aunai, and R. Smets, Phys. Plasmas 19, 022108 (2012).

[42] D. J. Southwood and M. G. Kivelson, J. Geophys. Res.-Space 98, 9181 (1993).

[43] L. Franci, P. Hellinger, L. Matteini, A. Verdini, and S. Landi, AIP Conf. Proc. 1720, 040003 (2016). 